\title{
“Le Voyage rétrospectif". Un texte important de Franz Hellens
}

\section{Marc Quaghebeur}

\section{(2) OpenEdition}

1 Journals

\section{Édition électronique}

URL : https://journals.openedition.org/studifrancesi/4547

DOI : 10.4000/studifrancesi.4547

ISSN : 2421-5856

Éditeur

Rosenberg \& Sellier

\section{Édition imprimée}

Date de publication : 1 avril 2012

Pagination : 99-109

ISSN : 0039-2944

\section{Référence électronique}

Marc Quaghebeur, " "Le Voyage rétrospectif". Un texte important de Franz Hellens », Studi Francesi [En ligne], 166 ( | LVI) | 2012, mis en ligne le 30 novembre 2015, consulté le 18 novembre 2021. URL : http://journals.openedition.org/studifrancesi/4547 ; DOI : https://doi.org/10.4000/studifrancesi.4547

\section{(c)}

Studi Francesi è distribuita con Licenza Creative Commons Attribuzione - Non commerciale - Non opere derivate 4.0 Internazionale. 


\section{"Le Voyage rétrospectif" Un texte important de Franz Hellens}

Comme son titre peut le laisser entrevoir, et comme l'indiquent les modalités de sa genèse - puisque le manuscrit est écrit, puis retravaillé après les faits -, Le Voyage rétrospectif ${ }^{1}$ de Franz Hellens (1881-1972) n'emmène pas ses lecteurs dans un carnet de voyage pittoresque tels qu'en ont produit maints voyageurs occidentaux de l'époque coloniale. Des villes et des sites sont pourtant évoqués, comme sont relatées des scènes de rues ${ }^{2}$, voire des rencontres.

Là n'est cependant pas le point de gravité de ces 81 pages manuscrites, relativement peu raturées, qui sommeillent dans les collections des «Archives \& Musée de la Littérature» à Bruxelles, sous la cote ML 2176. Elles se trouvent dans une farde préparée pour l'édition et comportant cinq autres textes dont un consacré au «fantastique réel». L'étiquette de la farde cartonnée porte l'adresse «rue de Naples 36» (la maison des enfants de l'écrivain qui leur avait été léguée par une romancière engagée, Neel Doff). L'auteur y vécut de 1948 à 1952. Le titre Le Voyage rétrospectif est inscrit entre la mention «articles - notes de voyages», qui est barrée, et des traits fortement raturés. À gauche, dans une écriture plus serrée, plus noire, on trouve: «doit être revu».

Si le narrateur-voyageur se déplace dans la Tunisie des années vingt, le propos de son récit vise un tout autre objectif: l'incarnation du «fantastique réel» dont Hellens se fit le chantre. Ce mot est celui par lequel Edmond Picard (1836-1924), le mentor des lettres belges francophones du XIX ${ }^{e}$ siècle, avait qualifié l'un des premiers textes d'Hellens, Les Hors-le-vent, dans 'La Chronique' du 18 décembre 1910. Pour Picard, le fantastique réel, ce n'est pas l'Invisible mais le «réel, vu, senti, en ses accidents énigmatiques, avec intensité». En quoi Picard ne faisait que prolonger ses propos de 1887 parus dans Le Juré sous le titre Le Fantastique réel.

Ces propos de Picard, d'avant 1914, paraissent décliner l'aventure d'Hellens dans son voyage en Afrique du Nord au milieu des années vingt: «Ce qui arrive, c'est ce à quoi on ne pense pas; tout arrive autrement qu'on ne le pensait, sinon complètement, du moins dans les détails».

Le Voyage rétrospectif est donc la remémoration par un homme mûr pris par très vieil homme du séjour qu'il fit en 1925 en Afrique du Nord, et la restitution-invention par ses mots d'un parcours initiatique rendu possible par la confrontation d'un Nordique avec un Orient apte à perturber les codes les mieux ancrés de sa perception. On retrouve donc dans ce texte des traces de l'orientalisme cher à deux siècles de

(1) Le texte a été édité en 2000 aux Presses universitaires Blaise Pascal de Clermont-Ferrand par Sourour Ben Ali, à partir du tapuscrit (ML 3336) issu des collections des AML et daté de mai 1966.

(2) L'édition de Sourour Ben Ali les commente, les remet en perspective historique, etc. 
fantasmes occidentaux. On peut tout autant y prélever des fragments d'anthologie restituant tel ou tel aspect de la société tunisienne de l'époque.

À chaque fois, le récit rebrasse cependant le poncif comme le détail folklorique ou réaliste pour l'amener à lui faire dire - très souvent en tous les cas - le contraire de ce qu'il est censé véhiculer. Ce dont il s'agit pour Hellens, c'est en effet de mener le lecteur vers ce qu'il cherche à lui faire entrevoir: l'au-delà de la rationalité occidentale au travers de détails du réel soigneusement mis en perspective. Un réel qui doit donc être décrit, y compris à travers les poncifs de sa perception littéraire et culturelle ${ }^{3}$ dominante. Hellens ne manque d'ailleurs pas de rappeler discrètement qu'il ne s'agit nullement pour lui, dans ce récit, de dupliquer Fromentin.

L'immersion dans une réalité aussi différente de l'Europe des années folles que la Tunisie d'avant le 9 avril 1934 constitue le creuset d'une métamorphose du sujet. Cela va conférer à la relation d'Hellens des aspects de voyage initiatique, sans jamais le transformer pour autant en récit mystique ou pédagogique. Telle est la singularité du paradoxe créateur contenu dans les mots de «fantastique réel». Les «réalités fantastiques» constituent une matière qu'Hellens avait par ailleurs choisi de donner à découvrir comme telle dans son recueil de nouvelles homonymes parues en 1923.

Le voyageur rétrospectif ne manque donc pas de pointer le caractère ondoyant de tout ce qu'il rencontre en Afrique sous l'aspect «le plus immuable» (p. 11). Et jusqu'à «l'homme qu'on dirait fait en série et ses constructions solides, rondes ou carrées, faites pour défier les siècles sous un ciel dévorant, mais dont la trame extérieure déroute par son dessin compliqué et ses tons chatoyants» (p. 22). Chaque mot de cette phrase véhicule tous les éléments de ce qui va induire et produire les rencontres d'Hellens en Afrique du Nord, comme la logique de la phrase qui les rapportera et les transposera.

Le récit suit une progression géographique qui mène le lecteur de la région de Tunis à Kairouan, avant de l'emmener à Constantine et Biskra, puis au désert. Telle est la trame réaliste, géographique, de la relation. Plus subtilement, la narration s'inscrit toutefois entre mer et désert - avec la connivence foncière et toujours double des éléments contenus dans ces deux espaces: la lumière, mais aussi l'adieu.

Entre eux, trois villes dessinent lieux ou contre-lieux de la révélation.

Constantine, d'une part. L'architecture du «conquérant» y a tracé «une grand'rue insignifiante dans son tracé et son air européen», tout à l'opposé du quartier chaud avec ses «ruelles tortueuses, montant en spirale ${ }^{4}$ vers on ne sait quels sommets chimériques» (p. 59). Les rues anciennes où les filles se laissent entrevoir à travers les «judas des portes ou derrière les barreaux des fenêtres» (p. 54) donnent à Hellens une sensation «d'hiératisme olfactif» (p. 59), mais aussi d'étrangeté purement arabe. Ces impressions sont telles qu'il désire regagner la grand'rue européenne et éprouve le sentiment d'avoir usurpé «un pouvoir, un droit» (p. 59).

L'effraction et l'éphémère sont constitutifs du fantastique réel on le sait.

Constantine fait contrepoint à Tunis dont la Médina a été préservée. C'est elle qui permet au voyageur un premier contact - déroutant - avec un monde radicale-

(3) La façon de faire d'Hellens n'a cependant rien à voir avec la violence du décalage qu'un Nougé (1895-1967) ou un Magritte (1898-1967), ses contemporains surréalistes, effectueront dans leurs œuvres à partir des codes usuels de la représentation culturelle.

(4) Métaphore qui caractérise aussi la musique arabe pour Hellens. 
ment autre. Un monde que le voyageur a tout d'abord nommé «l'Afrique», puis qu'il appellera «l'Arabie».

Cette globalisation indifférenciée de l'Autre absolu, typique du regard occidental de l'époque, ne suffit toutefois pas à cerner le propos d'Hellens. Ses généralisations s'avèrent en effet essentielles pour un propos qui entend se situer en dehors de l'historicité chère aux visions post-hégéliennes de l'Europe hégémonique - et d'autant plus que le narrateur a voyagé en ces contrées moins de sept ans après la fin de la boucherie de 1914-1918. Les six dernières nouvelles des Réalités fantastiques, parues en $1923^{5}$, y font d'ailleurs écho. Cette relation particulièrement tragique contient pourtant des notations qui constituent l'ingrédient du fantastique réel: la dimension d'éternité et d'immuable sur fond de vide spatial empli de bruits.

À la page 207 des Réalités fantastiques, Hellens ${ }^{6}$ écrit: «Le soleil baissait déjà. Jamais je n'avais regardé si longtemps le ciel, et je m'étonnais que dans ce grand vide, on pût découvrir tant de choses et que tout y prît la couleur de l'éternité». Rien d'étonnant dès lors à ce que dans une Tunisie métamorphosée en Afrique ou en Arabie, Hellens ait trouvé un espace accordé à ce qu'il recherche et dont il a besoin. L'écrivain privilégie dès lors ce qui renvoie tant à un en-deçà qu'à un présent immobile et éternel qui se révèle pour lui avec force dans les modes d'être du monde arabe.

La ville sainte de Kairouan dessine le troisième pôle urbain du livre dont elle constitue, très logiquement, le centre physique mais aussi métaphysique et narratif. La rencontre d'Hassan, jeune homme fortuné de la cité qui fit ses études en France, puis de sa sœur Fatima, amène en effet la seconde moitié du récit à plus de notations commentées que ce n'est le cas dans les quarante premières pages du manuscrit. Ces considérations sur la réalité humaine ethnicisée à laquelle se trouve confronté le voyageur constituent une autre dimension du récit d'Hellens.

Bien que ces passages puissent être isolés et intéresser qui cherche à connaître l'histoire de la Tunisie de l'époque, ils n'en font pas moins partie, tant d'abord, d'une architecture narrative symbolique bien différente. Celle-ci fonctionne au travers de discrets renvois d'images, tout sauf historiques, mais toutes vecteurs de rêve - et de rêve originaire qui plus est.

Aussi n'est-ce pas un hasard si le récit du voyageur n'accorde aucun intérêt aux vestiges romains qui abondent en Tunisie et s'écarte singulièrement de l'amour des ruines en vogue en Europe depuis le XVIII ${ }^{e}$ siècle. Hellens manifeste en revanche sa fascination pour Carthage dont il ne reste rien que le sable et la mer. Flaubert qui, selon lui, n'a donc rien vu au sens foncier - comme sa Salammbô - sont donc renvoyés par l'écrivain de Mélusine au rayon des antiquités et des bibeloteries dénuées d'intérêt. Car ce qui fascine Hellens dans Carthage, c'est précisément qu'elle n'est plus qu'un souvenir. Lieu voué «à l'oubli sans fin» (p. 12), elle est une civilisation dont «l'effacement sauvage vaut les plus belles survivances» (p. 27). Cette évocation l'emporte de loin pour l'écrivain belge sur l'obsession d'une reconstitution vériste à la Salammbô.

(5) Sous le titre générique Six réalités de guerre, Hellens publie six textes: «Premiers trophées»; «Breughel à l'Yser»; «La Fin de Nieuport»; «Sous l'abri»; «Fandor»; «Méditations devant la mer». À noter les majuscules d'une part, l'ancrage réaliste dans le front belge de la Première Guerre mondiale de l'autre (qu'Hellens n'a pas vécu mais visité).

(6) Ce sera également le cas d'un autre maître du réalisme magique, Paul Willems, durant la campagne des dix-huit jours en 1940. 
Le site maritime de la cité disparue permet donc à l'écrivain un basculement dans les éléments qui se trouve au cœur de toute sa démarche: «Rien que du sable, mais une plage émouvante par le dessin qui semble une grande initiale tracée dans la mémoire, où l'œil un peu sombre déborde, le mouvement des petites vagues et le grand soleil incandescent sur une rue lisse comme un rideau de soie» (p. 12). Ce promontoire marin, c'est celui «où naquit, fleurit, et s'écroula l'une des nations les plus réalistes et des plus fantasques du monde» (p. 12).

«Réaliste» et «fantasque», les deux mots qui structurent la singularité du récit sont bel et bien prononcés - et très vite - par l'auteur. Ils sont associés à la disparition du tangible comme à l'infini des sables auxquels conduisent ses diverses rencontres de la civilisation arabe. Celles-ci ne peuvent donc que s'achever dans le désert.

«Mer» et «sable», au début du récit. Sable et mouvance en sa fin. Avec Carthage comme point de fuite, et deux fois mise en abyme dans le texte.

Dans ce récit en mosaïque, marqué par l'emprise du rêve qui avance masqué et ne s'épand qu'à travers les mailles du réel, les conjonctions subtiles du réalisme et du fantastique ne se limitent pas aux allusions très personnelles d'Hellens à la cité punique. Le voyageur, qui se présente à plusieurs reprises comme un Nordique et ne manque pas de faire de nombreux renvois aux déperditions aquatiques et aériennes de la Norvège (où il s'est notamment rendu en 1934), affirme ainsi, dans la séquence «Fantasmes», qui précède celle qu'il a intitulée «Carthage»: «Si le Nord a ses fantômes, le Midi a ses fantasmagories. Là-bas, la nuit, ici le jour. Dans le Nord, le fantastique emprunte à la réalité. Ici la réalité prend l'apparence du fantastique» (p. 12).

Cette rencontre et cette évanescence, le narrateur ne va cesser de les relayer au gré de tableaux très secrètement enchâssés. La Tunisie (d'une époque révolue) aidant, ces tableaux descriptifs donnent à l'écriture d'Hellens une magie et une liberté que l'on trouve rarement dans ses textes, si ce n'est peut-être dans un autre récit rétrospectif ${ }^{7}$ consacré au portrait que fit de lui Modigliani à Nice en 1917.

La succession des tableautins du Voyage rétrospectif, qui empêche l'écriture d'Hellens de s'enliser dans les logiques discursives, fait alterner des saisies qui paraissent relever du récit de voyage, mais amarrent en fait le rêve au réel. Avec des passages dans lesquels la dérive vers la faille du songe se donne d'autant plus de champ d'expansion qu'elle s'est accrochée au préalable à des fragments de réel. La séquence «Tunis» est ainsi suivie d'une brève séquence «L'ordure». Celle-ci paraît correspondre aux clichés hygiénistes du Nord à l'égard du Sud censé être propice à la crasse . $^{2}$.

Après avoir cru se retrouver à Naples et avoir été confronté sur le paquebot à l'invasion de la «vermine des porteurs», Hellens bascule en réalité dans un univers au sein duquel une «atmosphère, odeurs, créatures humaines, et jusqu'aux pierres» (p. 3) lui donnent le sentiment d'une différence absolue. «La chaleur et l'animation sont ici deux choses nouvelles, qu'[il] ne saurai[t] pas encore définir - mais qu['il] pressen[t]» (p. 4). Elles le projettent loin des rives septentrionales de la Méditerranée.

(7) Modigliani, le voyant, texte publié à Bruxelles en 1986 aux Éditions Jacques Antoine, mais plus démonstratif.

(8) On trouve des traces du noyau dur de ce regard colonial, raciste et hygiéniste, chez E. PICARD, notamment dans El Moghreb al Aksa. Une mission belge au Maroc, publié chez F. Larcier en 1889 (avec des «interprétations» par le poète Théo Van Rysselberghe et un frontispice d'Odilon Redon), mais aussi dans la description des juifs du Maroc incluse par Picard dans sa Synthèse de l'antisémitisme (1892). 
Tunis, «c'est une autre lumière, poussiéreuse, directe et dure. D'autres ombres aussi, non moins dures mais bienfaisantes, on y va sans hésiter, et c'est là qu'il fait vraiment clair. Le reste est aveuglant. C'est de là aussi qu'on peut jouir de la vue des couleurs éblouissantes, non seulement des costumes, des objets, mais de l'odeur même» (p. 4).

Tout d'abord donc, un dépaysement - sans quoi la suspension qu'implique le fantastique réel est impossible. Une immersion ensuite dans le labyrinthique où l'ombre canalise la lumière d'une tout autre façon que dans le Nord. Une emprise olfactive enfin, qui va permettre toutes les synesthésies, y compris picturales.

En dehors de celles-ci, ne fonctionnerait pas l'émergence fugitive des réalités qui font fonctionner le fantastique réel. Le voyageur-narrateur se doit dès lors de les commenter, mais brièvement: «L'odeur. À Marseille, elle décorait le pavé, la rue; ici, elle fleurit l'homme» (p. 5 bis).

Aussi est-ce à partir des paradoxes induits par le cliché - parfois le plus apparemment choquant pour les sensibilités contemporaines - que l'écrivain opère ses métamorphoses.

Après avoir opposé, comme il le fait à maintes reprises dans Le Voyage rétrospectif, la Méditerranée européenne à celle de l'Afrique, Hellens, qui en est arrivé à constater que l'ordure prend en Afrique «figure humaine», parle de «saleté rayonnante». À se demander, écrit-il, si le plus étonnant, ce sont les «soies précieuses» et les «cuirs rouges»" sous le soleil ou bien les mendiants de rues «confondus avec l'ordure» (p. 5 bis).

Contraste magique, inducteur, et de l'écriture, et de sa révélation.Ce contrast amène en effet à un rapprochement, à une affirmation («Aucun doute») particulièrement décisifs, qui montrent bien l'ancrage imaginaire et culturel du fantastique réel. Comment le lecteur s'attendrait-il à voir évoquée alors la peinture de Rembrandt? «Dans l'éclairage artificiel ${ }^{10}$ de ses tableaux, Rembrandt avait deviné l'Orient. Il avait l'Orient en lui. Mais qui le lui avait donné? Comment, par quelle voie lui était-il venu? Étonnante rencontre du génie et de la réalité» (p. 5 bis).

Ce renvoi à l'une des figures majeures de l'un des arts dont l'Europe - et les anciens Pays-Bas en particulier - assura l'autonomisation à partir du XV siècle pourrait donner lieu à maints commentaires. Elle s'écarte du poncif méprisant de la saleté tout en y plongeant, et va dans les méandres de l'orientalisme pour restituer quelque chose de l'altérité sans vraiment y entrer. Tel est en somme l'effet recherché par Hellens.

Il inscrit en outre le travail de l'écrivain belge francophone dans la grande tradition par laquelle émergea, en tant que telle, une des premières littératures francophones. Et cela, même si Hellens jugea utile, pour s'affirmer, de s'opposer aux ténors de la génération précédente (Verhaeren, Maeterlinck); même s'il crut également bon de se battre en faveur d'une «littérature française de Belgique» censée ne manifester aucune singularité autre que celle d'un Tourangeau.

(9) Plus avant dans le récit, à Constantine, Hellens trace une séquence fort justement intitulée Deux Tableautins ce qui confirme l'extraordinaire construction du texte et la dissémination de ses indices autotéliques. Cette séquence commence par une scène de rues (descendantes) où le promeneur côtoie un mendiant d'une maigreur squelettique «et qui regardait le ciel». Cela lui paraît dessiner, autre métaphore plastique, une «espèce de "piéta" masculine» (p. 61). Ensuite, au sommet d'une rue (ascendante cette fois), le marcheur découvre sur un étal une pièce unique: «un portefeuille en cuir de bique, d'un beau rouge sang caillé, une chose fabriquée dans la solitude du désert, une de ces œuvres de patience et de solidité qui semblent faites pour l'éternité». Il l'achète et se hâte ensuite «de descendre la rue, sans se retourner» (p. 61).

(10) C'est moi qui souligne. 
Or le plus original de ce qu'écrit Hellens se situe dans la logique profonde de l'imaginaire et du travail singularisateur à travers lesquels des écrivains belges du $\mathrm{XIX}^{\mathrm{e}}$ siècle avaient fait émerger par rapport à la France une littérature francophone stricto sensu. La métaphore de la Flandre littéraire et picturale avait alors constitué le sésame de cette affirmation d'une littérature francophone distincte dans le contexte ambigu de l'affirmation des littératures nationales monolingues. Entre ses «transpositions d'art» et son écriture visuelle, Émile Verhaeren (1855-1916) avait par exemple consacré deux monographies majeures à deux peintres typiques à ses yeux de son espace d'origine: Ensor d'une part, Rembrandt de l'autre.

Dans Le Voyage rétrospectif, le clair-obscur cher au maître de La Ronde de nuit devient la clef d'une perception à laquelle mène le monde arabe tel qu'il apparaît à Hellens dans cette rétroaction mémorielle. Une rétroaction personnelle dont l'objectif n'a rien à voir avec le cadastre des civilisations.

Si le blanc - celui des bâtisses comme celui des tapis - est à ce point mis en valeur par le narrateur, c'est qu'il engendre des contrastes et produit les découvertes auxquelles s'attache l'écrivain. Les arts des villes arabes procurent donc une révélation comparable à celle des eaux et des sables. Ils induisent à un nouvel art de la fugue. Tout, dans la musique, l'art ou l'architecture arabes, n'est-il pas «fugue, éternel contrepoint, recherche inlassable d'un idéal qui fuit sans cesse, chassé par l'obsédant réalisme d'un climat et d'une terre qui entraînent à la paresse et obligent à l'action, où le rêve est une conquête de l'esprit sur le désoeuvrement ou l'automatisme des sens» (p. 30)?

Dans la logique de ce bref commentaire du Voyage rétrospectif, il ne me paraît pas essentiel de peser ou de soupeser les propos d'Hellens sur une civilisation, ni de s'appesantir sur le choix des réalités qu'il met en perspective - particulièrement dans la seconde partie du récit. Sa rencontre avec Hassan et Fatima à Kairouan, comme celle avec le chamelier du désert, ouvrent en effet à des propos que les historiens pourront par ailleurs extraire et analyser. Il s'agit en revanche ici de montrer une cohérence d'écriture que ce texte exemplifie de manière magistrale.

L'écriture s'appuie sur une réalité absolument exotérique pour mieux atteindre, à partir d'un détail ${ }^{11}$, et ainsi que l'avait judicieusement remarqué Edmond Picard, sa fantasmatique. En somme, c'est un détail du réel, souvent la reprise d'un motif exotique, qui permet de passer à l'exotérique sans tomber dans l'ésotérique.

Ce faisant, l'auteur restitue aussi une rencontre personnelle, mais qui n'est pas forcément interpersonnelle. C'est par elle que se décalent et se déroutent chez Hellens des ingrédients du récit de voyage orientaliste et colonial. Tremplin pour un voyage plus initiatique que touristique, le travail d'Hellens ne se donne pas pour mission première de révolutionner les canons de la logique narrative des récits qui ont accompagné ou préparé les expansions coloniales européennes.

Le voyageur sur le point d'accoster ne parle-t-il pas, comme je l'ai déjà indiqué, d'Afrique $^{12}-$ «Voilà l'Afrique, me dis-je avec admiration, et je sais que je ne verrai

(11) En route pour Kairouan, Hellens parle du fait que «certains détails offusquants d'une route toute proche [l] e rejetaient au large» (p. 36).

(12) Mélusine, roman d'Hellens paru en 1920 aux éditions de La Voile Rouge, débute par «En arrivant sur la côte d'Afrique, nous vîmes une foule de gens, aux costumes brillamment colorés, qui avançaient comme en pèlerinage». Cet imaginaire, antérieur, comme on le voit, au voyage tunisien proprement dit, n'est donc pas sans engendrer une part du Voyage rétrospectif. Hellens, qui a par ailleurs publié en 1922 chez Rieder Bass Bassina Boulou (l'histoire d'un fétiche africain); donna ensuite en 1923, aux éditions du Disque Vert, Réalités fantas- 
plus que des merveilles»-, (p. 3)? Son avancée dans le vieux royaume de Tunis se métamorphose ensuite et se spécifie bientôt en un parcours à travers l'Arabie.

Ces poncifs sont toutefois détournés et utilisés en vue de laisser (et de faire) advenir ce fantastique réel auquel des civilisations encore étrangères aux processus d'industrialisation qui s'emparent de plus en plus de la planète paraissent amener plus aisément que celles dont Hellens est issu. D'où le rapprochement effectué par l'écrivain entre le Grand Nord et le Désert; mais aussi ses jugements sévères sur «l'âpre cupidité du conquérant» (p. 60) occidental dont les réalisations urbanistiques sont évacuées d'un trait. Et cela, aussi bien lorsqu'il s'agit de la Tunis moderne «tout à fait ordinaire» (p. 4) ou de Constantine présentée comme un désastre. À la différence de Tunis, la vieille ville y subsiste en effet «où elle peut, démembrée, écartelée, par l'occupation européenne, fort peu respectée même dans ses vestiges le plus palpitants de vie authentique» (p. 59). L'Algérie n'est pas la Tunisie.

Les noms de pays ne sont jamais tracés par Hellens dans ce récit rétrospectif dans lequel le narrateur se laisse passer pour Français à Kairouan (p. 43), tout en renvoyant ailleurs explicitement aux plages belges de son enfance, La Panne ou Nieuport (p. 70). Là encore, il le fait cependant sans les rattacher au pays ${ }^{13}$. Cela fait partie des ambigüités et des chemins du fantastique réel, ce qui pourrait donner lieu à commentaires.

C'est en tous les cas par son potentiel encore inentamé de jeunesse et d'éternité (p. 18) que la Tunisie, cette Afrique/Arabie fille de Carthage, fascine Hellens et trame son récit rétrospectif. Et d'autant plus que le clair-obscur, métaphore picturale du fantastique réel, paraît avoir trouvé pour lui un répondant civilisationnel dans la culture arabe.

Le récit ne cesse de l'approcher au travers de l'architecture et de la musique. L'une et l'autre semblent, ainsi qu'Hellens le dit de la musique, «courir après ellesmêmes comme après une ombre» (p. 7). Ce déploiement ${ }^{14}$ au travers des contrastes d'un bâti dans lequel le blanc domine, dessine un perpétuel pas de deux avec les points de fuite de l'ombre, atteint sa majesté au crépuscule ${ }^{15}$. Cela permet à l'écrivain d'esquisser une synthèse de l'architectural et du musical, et de tracer quelques-unes des affirmations de son récit sur le caractère d'exception du monde dans lequel il est plongé. Cette fois, il s'agit du «soir arabe» (p. 11) et de son harmonie avec la voix du muezzin.

Façon de renvoyer au caractère foncièrement «ondoyant» mais premier $^{16}$ auquel l'Afrique sous son «aspect le plus immuable» (p.12) donne un accès privilégiée ${ }^{17}$.

tiques. La préface se termine par: «Je reconnus mes rêves. Je vis toute la nature qui leur faisait un cadre séduisant. Et je souhaitais de les voir toujours là, inaccessibles et libres, au ciel de l'antipode».

(13) L'analogie ne peut fonctionner intégralement, au plan de la dérive imaginaire, qu'à travers des espaces, et non des pays: «Est-il possible de juger du désert d'après ce que j’en ai vu, ce sable comme il en est sur toutes les plages, ces dunes si pareilles aux dunes de nos mers du Nord? N'était le chameau que je monte et l'ardeur volcanique du soleil, je pourrais me croire transporté sur le manteau de Sindbad dans les collines sablonneuses de La Panne ou de Nieuport» (pp. 69-70).
(14) Le blanc de lait des murs réverbère et repousse cette double obsession devenue unique. Elle frappe les yeux, la peau et pénètre jusqu'au cœur. «On ne pense plus, à peine sent-on encore, et cette espèce de sommeil du cerveau et des sens est sans rêve» (p. 14).

(15) «Il est à noter que Kairouan garde sa blancheur dans le crépuscule; et la nuit même, la lune absente, elle apparaît spirituelle et blanche dans sa robe de désert» (p. 57).

(16) «Le fatalisme musulman s'explique de luimême, vu sous l'angle de la puissance agonisante des éléments» (p. 17).

(17) Là aussi, sous un certain angle, on se 
Ingrédient indispensable du fonctionnement du fantastique réel, le «hasard» devient un des fils rouges de la description qu'Hellens trace des faits de vie - mariages inclus - en Tunisie. Les faits, il les commente en les reliant au génie spécifique de l'Afrique $^{18}$. Fréquent, le constat selon lequel «le fatalisme musulman s'incline devant la réalité» (p. 24) surgit ainsi au cœur de l'extraordinaire relation que l'écrivain donne $\mathrm{du}$ «Concert arabe». Cette généralisation, qui va de pair avec l'occultation des noms de pays - et donc, des histoires singulières - débouche sur une nouvelle maxime qui fonctionne sur le comparatisme interculturel. Le concert arabe est en effet pour Hellens un «exemple magnifique d'abnégation et de soumission à l'art dont on chercherait en vain l'équivalent en Europe» (p. 24).

Le Voyage rétrospectif comporte par conséquent une séquence consacrée au «Mystère d'une Race». Le texte y prend toutefois distance avec la supposée supériorité occidentale: «L'Européen a-t-il pris le temps de connaître cette grande famille nouvelle et de l'aimer [...]?» (p. 25). Hellens poursuit son propos et le renforce en mettant en exergue l'expérience de Lawrence. S'il le fait, c'est pour confesser que Lawrence lui-même estimait que «l'âme arabe lui est restée, malgré tout, impénétrable».

Le monde arabe peut donc permettre l'advenue du fantastique réel. La séquence ne s'achève-t-elle pas sur une sentence significative du dessein général du texte: «Les nuits étoilées d'Afrique sont pour l'âme ce que les journées ensoleillées sont pour les yeux. Elles incitent à veiller. L'âme aussi a besoin de réalité» (p. 25)?

Ces moments de veille/éveil, plus essentiels que le réel rencontré, donnent accès à la merveille. L'auteur les restitue et les magnifie au travers d'un jeu d'alternances, très finement maillées, de séquences descriptives et méditatives. Ainsi parvient-il notamment à revenir à sa hantise de la «combinaison ${ }^{19}$ des fugues» (p. 30). Tout n'est-il pas «miracle dans [s] on voyage» (p. 31)? Telle l'«image projetée sur un écran», une rue étroite et fermée prend donc forme et volume pour donner naissance à un subtil passage d'Hellens sur les fenêtres à colonnettes: «Cela finit, comme toute féerie, par une autre féérie, plus naturelle $[\ldots] \gg$ (p. 32).

Ce commentaire introduit à l'épisode du «Voile soulevé» par une belle Tunisienne, souvenir demeuré visiblement bien vivant chez l'écrivain du Voyage rétrospectif. Et comme il sied dans sa logique narrative, c'est ce «Voile soulevé» qui engendre stylistiquement la relation du trajet «Vers la ville sainte de Kairouan», l'auteur y confirme ainsi sa hantise du détail imprévu qui rend possible le fantastique réel. Cactus couverts de figues de Barbarie ou lacs salés dans la nuit laissent ainsi affleurer un fantastique du concret. «Terre de présent, ai-je dit de l'Afrique. C'est la première fois de ma vie que je n'ai pas attendu le rêve pour connaître la signification de ce mot positivement magique» (p. 37).

Comme Budapest ou Medina del Campo le furent également, à d'autres moments de la vie de l'écrivain belge, Kairouan constitue ${ }^{20}$ un des lieux majeurs de l'accomplissement du miracle à partir du vide; et la confirmation pour l'écrivain de l'adéquation de l'être arabe avec le «hasard heureux». L'arabe, peut donc écrire Hellens

trouve face à la reprise-transformation d'un motif récurrent du fantasme colonial. En même temps, il s'agit d'une réelle expérience littéraire en tous les cas.

(18) «[...] l'Afrique entière nous plie à la durée./ [...] Divine et tragique, voilà ce qu'elle est. [...]/
L'Afrique est la grande, l'absolue tragédie du présent $[\ldots] \gg($ p. 19).

(19) «Je me vois encore une fois obligé de recourir à la même comparaison» (p. 30).

(20) L'épisode se situe toutefois avant l'assomption due au désert. 
dans ce mélange de reprise transformatrice du poncif qui n'est qu'à lui, est «voleur. Pas voleur comme les autres. Voleur d'occasion» (p. 42). Dès lors, le pèlerin du fantastique réel ne peut que s'en sentir proche.

«L'architecture arabe» n'est-elle pas par excellence «une fugue, un labyrinthe d'où l'on s'évade vers l'infini» (p. 47)? Et la mosquée, non point un «temple consacré à Dieu, mais une construction où la Divinité vient» (p. 53)? Prudent mais cohérent, lucide également, Hellens tient en outre à préciser que sa perception choquera sans doute quelque savant connaisseur de l'Orient.

Lui se contente d'arriver en Tunisie «un peu à la façon du rêveur éveillé que la mémoire du présent oublie d'éclairer» (p. 53).

Faut-il en dire plus de ce texte singulier, aussi étranger aux relations de voyage qu'aux récits initiatiques, et qui entend précisément se situer aux intersections du réel et du songe? Sinon qu'il s'inscrit dans un après-coup du romantisme. Le texte l'évoque dès sa deuxième page, mais aussi dans une singulière continuité (bien sûr non dite, ce qui est typiquement belge) avec la perpétuation de l'emprise picturale ${ }^{21}$ et de l'obsession de l'entre-deux par lesquelles cette littérature francophone s'était définie dès le XIX ${ }^{e}$ siècle.

Il ne s'agit pas pour autant de limiter l'approche d'un tel Voyage rétrospectif au seul fil rouge du fantastique réel mais d'en expliquer la singularité. L'on trouve, par exemple, dans ce récit des réflexions sur l'évolution sociale qui ne sont pas sans rapport avec cette problématique de l'entre-deux. Hellens les met à l'enseigne de la métaphore de la rencontre (ou non?) de la caravane et du cargo ${ }^{22}$. Ses considérations sur le concert arabe ou sur les tapis de Kairouan, sur la palmeraie, «ce miracle de l'eau» (p. 65), comme sur l'amphore concourent toutefois à la mise en exergue, dans l'apparemment immuable, de la faille momentanément salvatrice qui fait basculer, sans délirer pour autant, dans l'en-deçà et l'au-delà du rationnel occidental.

Tout cela se joue à travers de subtils renvois intra-textuels. Ainsi ceux qui vont $\mathrm{du}$ «Voile soulevé» des deux belles de Tunis (au début du récit) au «voile de verre fondu» (p. 34) que le sirocco tresse autour du visage du voyageur perdu en plein désert, éperdu de vide et d'infini limité, que l'on trouve à la fin du récit. Entretemps, l'écrivain a fait passer le lecteur par une discussion du narrateur sur le voile des femmes, discussion qu'il a avec Fatima, sœur d'Hassan, laquelle s'est présentée dévoilée devant lui, à Kairouan. À elle seule, cette indication interdit toute autonomisation de la fantasmagorie.

«Le fait arabe - écrit Hellens dans le chapitre Biskra - n'est peut-être qu'une perpétuelle hantise du désert, du vide qui attire, et dont le nom seul est un caractère, un idéal, mais aussi une perpétuelle désillusion» (p. 67). Ceux du fantastique réel à maints égards.

(21) Parlant - fort bien - de l'amphore qui pour Hellens est la forme même de l'Orient, l'écrivain rappelle, apparemment incidemment, que son «enfance a été dominée par les images de Gustave Doré illustrant une Bible de distribution des prix» (p. 52). Des femmes y revenaient de la rivière, «l'urne sur la tête». J'ai montré ailleurs, à propos de l'Afrique centrale, comment ce même motif engendrait chez Léopold Courouble, alors juge des palabres dans le Bas-Congo, une description essentielle à sa fantasmagorie et à sa perception de l'autre.

(22) «Un peuple installé sur le double appui du passé et du présent, demeuré fort dans sa foi ancienne, sa conviction et son ardeur actuelles, se laissera-t-il éternellement subjuguer par une civilisation qui ne veut tenir compte que du quotidien profitable, et pour qui l'avenir n'est qu'une répétition plus perfectionnée du présent?» (p. 60). 
L'origine de l'obsession et de l'exacerbation du fantastique réel dans Le Voyage rétrospectif reste à creuser. Peut-être faut-il la mettre en rapport avec les hantises d'un homme de la Première Guerre mondiale.

Pourquoi Hellens, au-delà de son souci - très «littérature française de Belgique»-de biffer ses origines, a-t-il en effet jugé nécessaire en 1931 (moment de la nouvelle édition chez Gallimard ${ }^{23}$ de ses Réalités fantastiques) de gommer les six nouvelles relatives aux années 1914-1916? Ancrées dans une réalité précise et donc indépassable, où l'universalisation par la métaphore analogique ne peut être menée à terme, elles expliquent sans doute l'origine de l'exacerbation de ce besoin de fantastique réel qui s'empare des lettres belges de l'époque. Robert Poulet (1902-1989), par exemple, fut un héros des combats de 1914-1918 avant de sombrer en 1940 dans un fascisme national. Il fut, lui aussi, non seulement un adepte et un défenseur du fantastique réel, mais aussi de la notion de littérature française de Belgique. N'entre-t-il pas sur la scène littéraire, en 1931, avec Handji qui se déploie sur l'immobilité des confins perdus du front de Galicie, mais n'a rien à voir avec un récit de guerre?

Comme les nouvelles de guerre retirées des premières Réalités fantastiques, le livre projeté par Hellens en 1966 devait comporter six textes. Parmi ceux-ci, un «Claudel en Belgique» et une «Célébration de la pomme», mais également un texte sur le fantastique réel. Cela ne laisse pas d'interroger. Et d'autant plus que Le Voyage rétrospectif constitue une des incarnations les plus réussies et les plus déliées de l'œuvre d'Hellens.

La rétractation devant la publication de texte fait de tableautins enchâssés, application parfaite et exceptionnelle de l'esthétique du détail, provient-elle de l'obsession de la phrase classique française qui n'a pas toujours servi l'œuvre d'Hellens? Et/ou de son malaise face à l'usage de motifs de description du monde arabe, usuels avant les Indépendances, mais qu'il croit alors difficile de maintenir?

Reste que Le Voyage rétrospectif est une des œuvres d'Hellens qui comporte la trace d'une blessure d'humanité antérieure au voyage et que, sans cette blessure, l'esthétique du fantastique réel n'aurait vraisemblablement pu être menée à terme - à son autonomisation.

Sans doute est-ce une des conséquences singulières des deux guerres civiles européennes du $\mathrm{XX}^{\mathrm{e}}$ siècle, indûment prétendues mondiales?

Hasard aussi, le fait que ce soit au désert ${ }^{24}-$ celui des sables - comme à Carthage disparue qu'Hellens confie le soin de ses métamorphoses salvatrices? Je ne le pense pas.

L'affleurement du fantastique réel, qui connut en Belgique jusqu'aux années 1980 une emprise assez unique, présuppose une immersion dans un monde que l'Histoire présente ne marque pas de son sceau. Dans une Tunisie aujourd'hui disparue, et sans doute mythifiée, Hellens paraît avoir trouvé un des lieux les plus propices à son épanouissement. D'elle, il nous restitue cependant quelque chose d'important.

(23) Il y reprend en revanche Les Hors-le-vent (1909) qu'avait commentés Picard.

(24) «Ce que l'Arabe fait, pense, proclame, dé- sire, demandez-le au désert tout proche. Mais essayez d'abord de comprendre le désert» (p. 68). 
Le fantastique réel pour dépasser l'impossibilité d'une Histoire humanisée?

L'écrivain conclut en tous les cas son livre en des termes importants pour qui veut entendre l'enjeu de son texte: «Je n'oublierai pas le sourire de Mohammed. Il y a donc des hommes, comme des bêtes ${ }^{25}$, à la mesure du désert» (p. 81).

MARC QUAGHEBEUR

(25) «Comme des bêtes» constitue un ajout dans le manuscrit. 